\title{
Instillation Method of Administration
}

National Cancer Institute

\section{Source}

National Cancer Institute. Instillation Method of Administration. NCI Thesaurus. Code C149596.

The technique of administration of a pharmaceutical product drop by drop onto or into the affected area. 